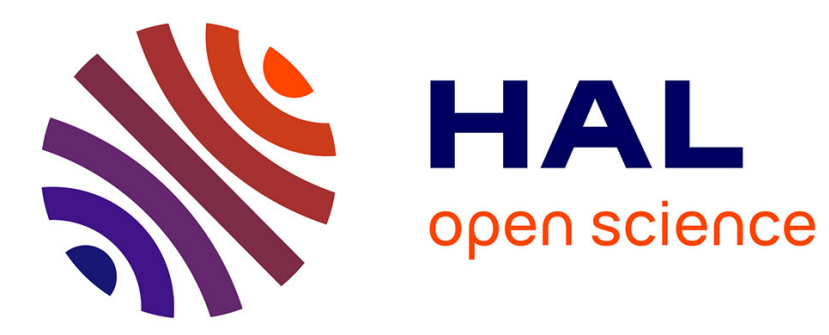

\title{
Analytical results for solar sail optimal missions with modulated radial thrust
}

Alessandro A. Quarta, Giovanni Mengali

\section{To cite this version:}

Alessandro A. Quarta, Giovanni Mengali. Analytical results for solar sail optimal missions with modulated radial thrust. Celestial Mechanics and Dynamical Astronomy, 2010, 109 (2), pp.147-166. 10.1007/s10569-010-9319-x . hal-00595947

\section{HAL Id: hal-00595947 https://hal.science/hal-00595947}

Submitted on 26 May 2011

HAL is a multi-disciplinary open access archive for the deposit and dissemination of scientific research documents, whether they are published or not. The documents may come from teaching and research institutions in France or abroad, or from public or private research centers.
L'archive ouverte pluridisciplinaire HAL, est destinée au dépôt et à la diffusion de documents scientifiques de niveau recherche, publiés ou non, émanant des établissements d'enseignement et de recherche français ou étrangers, des laboratoires publics ou privés. 


\title{
Analytical Results for Solar Sail Optimal Missions with Modulated Radial Thrust
}

\author{
Alessandro A. Quarta · Giovanni Mengali \\ the date of receipt and acceptance should be inserted later
}

Keywords Solar Sail, Radial Thrust, Optimal Trajectories.

\begin{abstract}
The aim of this paper is to analyze the optimal trajectories of a spacecraft subjected to a modulated radial thrust, whose magnitude is inversely proportional to the square of the distance from the primary body. This case is representative of a Sun-facing solar sail with a passive attitude control system. In this study the sailcraft is assumed to perform a finite number of reorientation maneuvers to set the propelling acceleration to zero and generate suitable coasting arcs along the trajectory. Accordingly, the resulting generalized orbit is a sequence of either propelled or ballistic conic arcs, whose main characteristics (in terms of semimajor axis, eccentricity, and perihelion radius) can be calculated in closed form. As a result, the sailcraft optimal performance can be studied using an analytical approach. In particular, some compact relationships are drawn and discussed that allow one to find the optimal sailcraft characteristics required to reach a prescribed final orbit.
\end{abstract}

\section{Introduction}

The trajectory design of a solar sail based mission is usually addressed in an optimal framework (Dellnitz et al., 2009; Racca, 2003). Because the solar sail uses no propellant, the aim of the optimization process is to find suitable relationships between the sail performance, as the maximum propelling acceleration at

A. A. Quarta · G. Mengali

Dipartimento di Ingegneria Aerospaziale, University of Pisa, Via G. Caruso 8, I-56122 Pisa, Italy

A. A. Quarta E-mail: a.quarta@ing.unipi.it·G. Mengali, corresponding author, E-mail: g.mengali@ing.unipi.it 
launch, and the total flight time. Apart from the mathematical approach used to tackle the problem (Betts, 1998), the results of an optimal analysis are generally obtained in numerical form. In fact, in most cases the solar sail trajectory cannot be computed analytically, as it arises from a fairly involved optimal steering law (Mengali and Quarta, 2005c; Mengali et al., 2007).

The propelling acceleration direction and magnitude of a solar sail depend on the sail attitude and on the sailcraft distance from the Sun. However, when the sail attitude is held fixed, the propelling acceleration direction is a constant of motion in an orbital frame, whereas its magnitude changes with the distance from the Sun in the same way as the solar radiation pressure does. If one neglects the potential anisotropy in the Sun's irradiation (McInnes and Brown, 1990), and assumes that the sail nominal plane is normal to the incoming sunrays, the propelling acceleration is, in its turn, purely radial, with a magnitude directly proportional to the local gravitational pull.

The motion of a constant mass spacecraft under a radial thrust inversely proportional to the square of the distance from the primary body has been studied by Boltz (1991), and then further investigated by McInnes (2003) and Yamakawa (2006). From a practical point of view, a pure radial thrust can be obtained with a passive control by means of a sail having a slightly conical form, and whose apex is directed sunward (McInnes, 2003; Mengali and Quarta, 2005b). The use of a passive control system provides a substantial simplification to the whole mission design process, because it avoids the complex task of continuously varying the sail attitude, which represents one of the most challenging mission goals (Kirpichnikov et al., 1995; Koblik et al., 2003). In most cases, however, a passive control is too restrictive as it does not guarantee the fulfilment of some minimum performance criteria. In fact, the use of a Continuous Radial Thrust (CRT) provides unnecessarily conservative results in terms of required maximum thrust level to accomplish a given mission. Not surprisingly, some compromise solutions are often adopted as, for example, the use of a piecewise-constant steering law (Otten and McInnes, 2001; Mengali and Quarta, 2009c).

In this paper a different compromise solution is chosen. More precisely, it is assumed that the sailcraft attitude motion is purely passive with the exception of a finite number of reorientation maneuvers whose aim is to rotate the sail such as to set the thrust to zero and generate suitable coasting arcs along the trajectory, see Figure 1. 


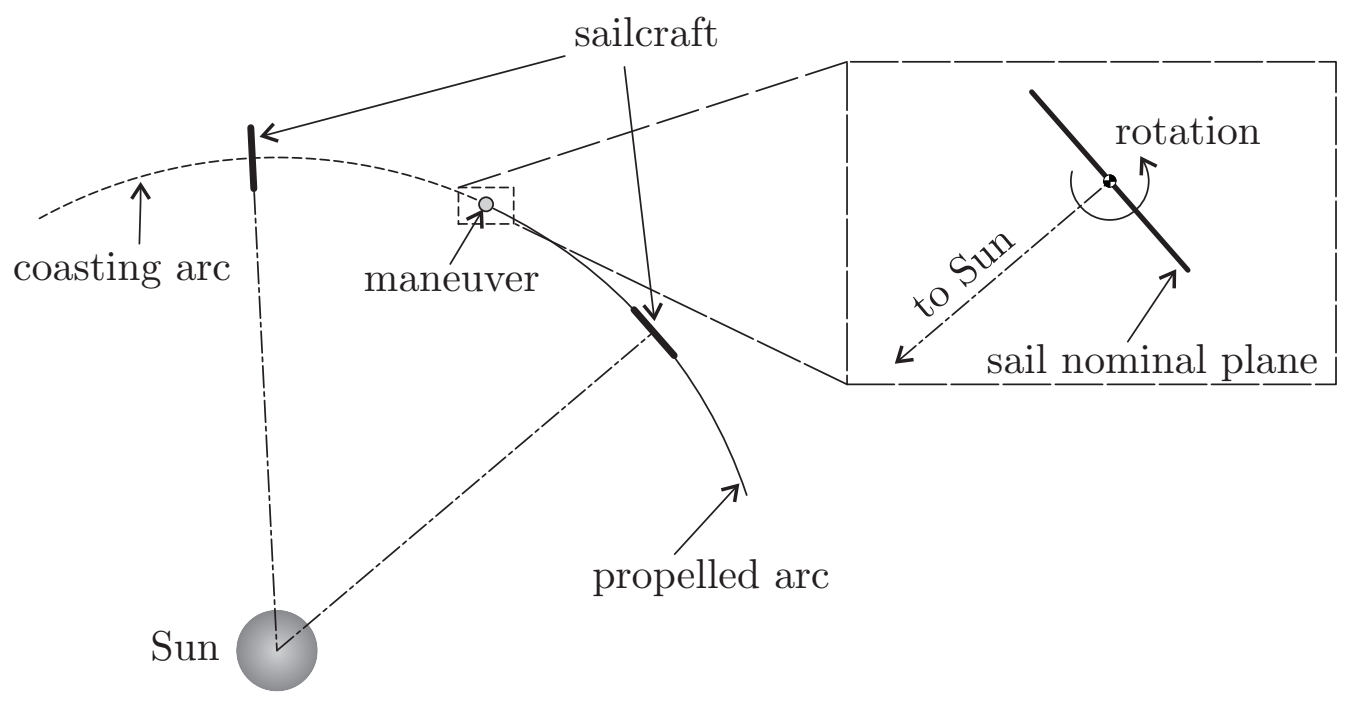

Fig. 1 Sailcraft orientation during a propelled arc (solid line) and a coasting arc (dashed line).

Each maneuver is nearly instantaneous, as the time interval necessary to complete the sail reorientation is negligible with respect to the characteristic mission time. Such a Modulated Radial Thrust (MRT) strategy, applied at a constant-thrust-level propulsion system, has been recently investigated by Quarta and Mengali (2009), who have shown that a MRT guarantees a substantial reduction in the performance required to fulfil a given mission when compared to a CRT case. The aim of this paper is to extend the analysis of Quarta and Mengali (2009) to a solar sail spacecraft, whose propelling acceleration is modulated and varies as the inverse square distance from the Sun. The resulting sailcraft trajectory is a sequence of either propelled or ballistic conic arcs. The peculiarity of such a trajectory allows one to calculate analytically the characteristics of each conic arc, in terms of both eccentricity and semimajor axis length. In addition, for a given geometry of the initial sailcraft orbit and a given value of solar sail lightness number $\beta$ (the ratio of the maximum solar radiation pressure acceleration to the solar gravitational acceleration), it is shown that the geometrical characteristics of any conic arc depend only on the distances at which the reorientation maneuvers are performed. As a result, for a given value of $\beta$ and an assigned number of mission arcs, it is possible to calculate the sequence of reorientation maneuvers that optimize a given performance index.

Firstly, the strategy that maximizes the semimajor axis length of the final propelled arc is studied. It is shown that the corresponding control law consists in setting the thrust on as long as the sail moves away from the Sun, and setting it to zero when the sailcraft approaches the Sun. As a special case of 
the above strategy, the escape problem for a propelled trajectory is investigated. It is well known that, for a solar sail subjected to a CRT, there exists a minimum value of lightness number $\beta_{\text {min }}$ below which the sail cannot attain an escape condition (McInnes, 2003). Using an optimal MRT strategy the escape condition can be obtained even if $\beta$ is substantially less than $\beta_{\min }$. Moreover, some simple analytical relationships are found that link the number of trajectory arcs with the minimum necessary value of $\beta$, the total mission time, and the minimum perihelion distance.

As a second mission scenario, the minimum value of $\beta$ required to reach a ballistic arc with a given value of semimajor axis is studied. The resulting optimal control law turns out to be the symmetric counterpart of the previous steering law, that is, the sail thrust is on when the sailcraft approaches the Sun and is off when it moves away from it. This steering law is applied to study flyby missions to outer planets and to generate Earth resonant Keplerian orbits.

\section{Mathematical Model}

Consider a solar sail spacecraft moving in an elliptic parking orbit with semimajor axis $a_{0}$ and eccentricity $e_{0}$. Assume that the solar sail provides a pure radial thrust whose magnitude is

$$
a_{r}=\beta \frac{\mu_{\odot}}{r^{2}}
$$

where $\mu_{\odot}$ is the Sun's gravitational parameter and $r$ is the Sun-sailcraft distance. The value of the lightness number $\beta<1$ quantifies the sailcraft performance and is a function of the sail reflecting area, the total spacecraft mass and the optical properties of the reflective film (Wright, 1992; Mengali et al., 2007).

An equivalent parameter that can be used to quantify the performance of a solar sail is the characteristic acceleration $a_{c}$, i.e., the maximum propelling acceleration at 1 AU distance from the Sun. Substituting $r=1$ AU into Eq. (1), yields

$$
a_{c} \simeq(5.93 \beta) \mathrm{mm} / \mathrm{s}^{2}
$$

When subjected to a pure radial thrust, the sailcraft trajectory describes a generalized orbit, that is, an orbit obtained through a modulated, inverse square thrust (McInnes, 2003; Mengali and Quarta, 2007). The resulting trajectory is actually a conic, as the sailcraft moves under the effect of a gravitational 
force whose magnitude is reduced with respect to the solar gravity. In addition, the sailcraft propulsion is switched off at some points along the trajectory, by suitably re-orienting the solar sail, to create a sequence of mission phases, characterized by either a propelled or a coasting arc, as schematically shown in Fig. 2. By assumption the passage P-C from a propelled arc to a coasting arc (or, viceversa C-P, from a

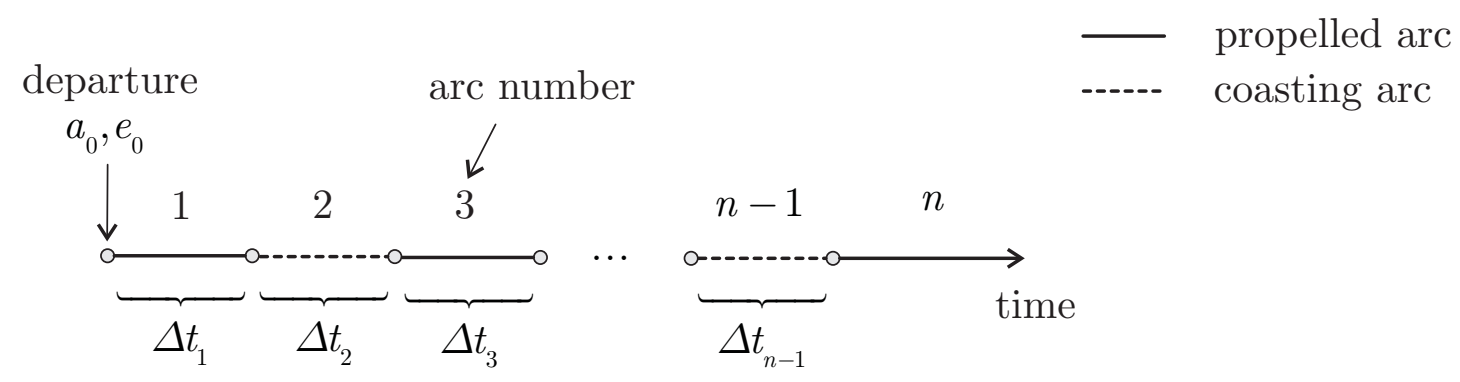

Fig. 2 MRT mission strategy for a $n$-arc trajectory ( $\Delta t_{k}$ is the $k$-th arc length).

coasting to a propelled arc) is performed with an instantaneous reorientation maneuver, thus implying a continuity in both the sailcraft position and velocity just before and after the maneuver accomplishment.

During a propelled arc the sailcraft describes a two-body motion subjected to a reduced gravitational parameter $\widetilde{\mu}_{\odot}$ given by (McInnes, 2003)

$$
\widetilde{\mu}_{\odot} \triangleq \mu_{\odot}(1-\beta)
$$

Consider now a mission constituted by a total of $n$ distinct arcs, and let $k$ be the generic mission arc. With the symbology of Fig. 2, $k=0$ corresponds to the starting sailcraft orbit with eccentricity $e_{0}$ and semimajor axis $a_{0}$, while $k=1$ is the first propelled arc. Consider the problem of finding the value of $a_{1}$, that is, the semimajor axis of the orbit in the first phase. Let $r_{0} \in a_{0}\left[\left(1-e_{0}\right),\left(1+e_{0}\right)\right]$ be the sailcraft distance at which the maneuver is performed. From the mechanical energy equation one obtains:

$$
\mu_{\odot}\left(\frac{1}{r_{0}}-\frac{1}{2 a_{0}}\right)=\widetilde{\mu}_{\odot}\left(\frac{1}{r_{0}}-\frac{1}{2 a_{1}}\right)
$$

Substituting Eq. (3) into (4), and solving for $a_{1}$ yields:

$$
a_{1}=\frac{a_{0}(1-\beta)}{1-2 a_{0} \beta / r_{0}}
$$


Because $2 a_{0} / r_{0}>1$, Eq. (5) implies that a C-P maneuver increases the semimajor axis (that is, $\left.a_{1}>a_{0}\right)$.

The orbit eccentricity $e_{1}$ of the first arc is obtained by observing that a radial thrust does not change the magnitude of the sailcraft angular momentum $h$. In fact, from $h=\sqrt{\mu_{\odot} p_{0}}=$ constant, one obtains:

$$
\mu_{\odot} p_{0}=\widetilde{\mu}_{\odot} p_{1}
$$

or

$$
p_{1}=\frac{p_{0}}{1-\beta}
$$

The orbit eccentricity is, therefore

$$
e_{1}=\sqrt{1-\frac{p_{0}}{a_{1}(1-\beta)}}
$$

From Eqs. (5) and (8) the orbital characteristics of the first arc are closely related to the value of $\beta$ and to the distance $r_{0}$ at which the maneuver is performed. Consider now the second arc $k=2$ (a coasting arc, see Fig. 2) and assume that the second maneuver is performed at a solar distance $r_{1} \geq a_{1}\left(1-e_{1}\right)$. From the energy equation, the relationship between $a_{2}$ and $a_{1}$ is:

$$
a_{2}=\frac{a_{1}}{1+\beta\left(2 a_{1} / r_{1}-1\right)}
$$

In this case, because $2 a_{1} / r_{1}>1$, a P-C maneuver implies a reduction of the semimajor axis $\left(a_{2}<a_{1}\right)$. Substituting Eq. (5) into (9), the semimajor axis can be expressed as a function of the parking orbit characteristics $\left(a_{0}\right)$ and of the sailcraft system $(\beta)$. The result is:

$$
a_{2}=\frac{a_{0}}{1-2 a_{0} \beta\left(\frac{1}{r_{0}}-\frac{1}{r_{1}}\right)}
$$

Equation (10) shows that $a_{2} / a_{0}$ can be either positive or negative, depending on the magnitude of the second term in the denominator (the latter is a function of $r_{0}$ and $r_{1}$, that is, of the points at which the two maneuvers are performed). From the angular momentum conservation one has

$$
(1-\beta) p_{1}=p_{2}
$$


Recalling Eq. (7) one obtains

$$
p_{2}=p_{0}
$$

from which

$$
e_{2}=\sqrt{1-\frac{p_{0}}{a_{2}}}
$$

where $a_{2}$ is given by Eq. (10).

The generalization to a generic $k$ (with $k=1,2, \ldots, n$ ) is straightforward, because the expressions for the semimajor axis $a_{k}$, the semilatus rectum $p_{k}$, and the eccentricity $e_{k}$ of the $k$-th arc depend only on whether $k$ is odd (propelled arc) or even (coasting arc). More precisely, one has

$$
\begin{gathered}
a_{k}= \begin{cases}\frac{a_{0}(1-\beta)}{1-2 \beta a_{0} F_{k}} & \text { if } k \text { is odd } \\
\frac{a_{0}}{1-2 \beta a_{0} F_{k}} & \text { if } k \text { is even }\end{cases} \\
p_{k}= \begin{cases}\frac{p_{0}}{1-\beta} & \text { if } k \text { is odd } \\
p_{0} & \text { if } k \text { is even }\end{cases}
\end{gathered}
$$

and

$$
e_{k}= \begin{cases}\sqrt{1-\frac{p_{0}}{a_{0}(1-\beta)^{2}}\left(1-2 \beta a_{0} F_{k}\right)} & \text { if } k \text { is odd } \\ \sqrt{1-\frac{p_{0}}{a_{0}}\left(1-2 \beta a_{0} F_{k}\right)} & \text { if } k \text { is even }\end{cases}
$$

where

$$
F_{k} \triangleq \sum_{i=0}^{k-1} \frac{(-1)^{i}}{r_{i}}
$$

Note that for a given $a_{k}$, the corresponding $F_{k}$ is obtained from Eq. (14). Therefore, from Eq. (16), the eccentricity $e_{k}$ depends only on $\beta$ and $a_{k}$. This result is not at all surprising, as it arises implicitly from the conservation of the angular momentum $h$. Using the polar equation of a conic section, an equivalent expression for $F_{k}$ is given by:

$$
F_{k}=\sum_{i=0}^{k-1} \frac{(-1)^{i}\left(1+e_{i} \cos \nu_{i}\right)}{p_{i}}
$$


where $\nu_{i}$ is the sailcraft true anomaly at the maneuver point. In a $n$-arc mission, Eqs. (14) and (16) can be used to find the sailcraft performance (in terms of lightness number $\beta$ ) required to reach an orbit of given characteristics. In fact, the values of $a_{n}$ and $e_{n}$ of the final orbit depend both on $\beta$ and on the summation in Eq. (18) (with $k=n$ ). The latter, in its turn, depends on the points $\left(\nu_{i}\right)$ at which the maneuvers are executed. Therefore, the values $\nu_{i}$ constitute a set of $n$ control parameters whose choice defines the final value of $a_{n}$ and $e_{n}$ through Eqs. (14) and (16). In particular, an interesting class of trajectories is found by looking for the sequence of maneuvers that either maximize or minimize $F_{n}$. For example, as shown later, the maximization of $F_{n}$ is useful for calculating the minimum value of $\beta$ that allows a sailcraft to reach an escape condition after $n$ arcs. In principle such a problem could be tackled by looking for the extrema of a function of $n$ independent variables. For example, from Eq. (17) the maximum of $F_{n}$ is obtained by performing the maneuver when the Sun-sail distance $r_{i}$ is at a minimum (maximum) when $i$ is even (odd). By symmetry, the minimum of $F_{n}$ is obtained when $r_{i}$ is at a maximum (minimum) if $i$ is odd (even). The same result can also be obtained following a different approach, with the aid of the calculus of variations. This allows one to introduce the mathematical model that will be used in the trajectory simulations. The details are discussed in the next section.

\section{Sequence of optimal maneuvers}

The problem of minimizing or maximizing the function $F_{n}$ of Eq. (18) is now investigated using an indirect approach. To this end, introduce the sailcraft equations of motion in vector form, viz.

$$
\begin{aligned}
\dot{\boldsymbol{r}} & =\boldsymbol{v} \\
\dot{\boldsymbol{v}} & =-\frac{\mu_{\odot}}{r^{3}} \boldsymbol{r}+\tau \beta \frac{\mu_{\odot}}{r^{3}} \boldsymbol{r}
\end{aligned}
$$

where $\boldsymbol{r}$ and $\boldsymbol{v}$ are the sailcraft position and velocity vectors (with $r \triangleq\|\boldsymbol{r}\|$ ), and $\tau=(0,1)$ is the switching function $(\tau=1$ when the sail nominal plane is perpendicular to the incoming sunrays, and $\tau=0$ during a coasting arc) that models the reorientation maneuver.

Consider first the problem of optimizing $F_{n}$. This amounts to looking for the steering law $\tau=\tau(t)$ that maximizes (or minimizes) the specific mechanical energy $\mathcal{E}$ at a given final time instant $t_{f}$. The 
Hamiltonian associated to the equations of motion is:

$$
H \triangleq \boldsymbol{\lambda}_{r} \cdot \boldsymbol{v}-\frac{\mu_{\odot}}{r^{3}} \boldsymbol{\lambda}_{v} \cdot \boldsymbol{r}+\tau \beta \frac{\mu_{\odot}}{r^{3}} \boldsymbol{\lambda}_{v} \cdot \boldsymbol{r}
$$

where $\boldsymbol{\lambda}_{r}$ and $\boldsymbol{\lambda}_{v}$ are the vectors adjoint to $\boldsymbol{r}$ and $\boldsymbol{v}$, respectively. Their time derivatives are given by the Euler-Lagrange equations (Bryson and Ho, 1975; Kim and Hall, 2005), that is:

$$
\begin{aligned}
& \dot{\boldsymbol{\lambda}}_{r}=-\frac{\partial H}{\partial \boldsymbol{r}}=-\frac{2 \mu_{\odot}(1-\tau \beta)}{r^{3}} \boldsymbol{\lambda}_{v} \\
& \dot{\boldsymbol{\lambda}}_{v}=-\frac{\partial H}{\partial \boldsymbol{v}}=-\boldsymbol{\lambda}_{r}
\end{aligned}
$$

The two first order differential equations (22) and (23) can be merged into a single second order differential equation involving the primer vector $\boldsymbol{\lambda}_{v}$ alone (Lawden, 1963):

$$
\ddot{\boldsymbol{\lambda}}_{v}=\frac{2 \mu_{\odot}(1-\tau \beta)}{r^{3}} \boldsymbol{\lambda}_{v}
$$

Bearing in mind the equations of motion (19)-(20), the solution of Eq. (24) is (Lawden, 1963):

$$
\lambda_{v}=c \boldsymbol{v}
$$

where $c \neq 0$ is a constant parameter. The sign of $c$ depends on the transversality condition. When the problem is to maximizing the final specific mechanical energy $\mathcal{E}$, the transversality condition is (Bryson and Ho, 1975; Stengel, 1994):

$$
\left.\boldsymbol{\lambda}_{v}\right|_{t_{f}}=\left.\frac{\partial \mathcal{E}}{\partial \boldsymbol{v}}\right|_{t_{f}}=\left.\boldsymbol{v}\right|_{t_{f}}
$$

If, instead, the control problem aims at minimizing the value of $F_{n}$, (equivalently, minimizing $\mathcal{E}$ at a given $\left.t_{f}\right)$, the transversality condition becomes:

$$
\left.\boldsymbol{\lambda}_{v}\right|_{t_{f}}=-\left.\frac{\partial \mathcal{E}}{\partial \boldsymbol{v}}\right|_{t_{f}}=-\left.\boldsymbol{v}\right|_{t_{f}}
$$


Comparing Eqs. (25), (26) and (27), one concludes that the primer vector satisfies the following relationships:

$$
\boldsymbol{\lambda}_{v}=\left\{\begin{array}{lll}
\boldsymbol{v} & \text { if } & F_{n}=F_{n}^{\max } \\
-\boldsymbol{v} & \text { if } & F_{n}=F_{n}^{\min }
\end{array}\right.
$$

where $F_{n}^{\max }\left(F_{n}^{\min }\right)$ is the maximum (minimum) value of $F_{n}$.

We are now in a position to obtain the optimal steering law as a function of the primer vector direction. From Pontryagin's maximum principle, the optimal control law $\tau(t)$ is such that, at any time, the Hamiltonian $H$ of Eq. (21) is an absolute maximum. Because $H$ is linear in the control variable $\tau$, a bang-bang control law is optimal (Stengel, 1994). According to Lawden (1963) the result is:

$$
\tau=\left\{\begin{array}{ll}
1 & \text { if } \quad \boldsymbol{v} \cdot \boldsymbol{r}>0 \\
0 & \text { if } \quad \boldsymbol{v} \cdot \boldsymbol{r}<0
\end{array} \quad \text { when } \quad F_{n}=F_{n}^{\max }\right.
$$

and

$$
\tau=\left\{\begin{array}{ll}
0 & \text { if } \quad \boldsymbol{v} \cdot \boldsymbol{r}>0 \\
1 & \text { if } \quad \boldsymbol{v} \cdot \boldsymbol{r}<0
\end{array} \quad \text { when } \quad F_{n}=F_{n}^{\min }\right.
$$

The previous relationships state that when $F_{n}=F_{n}^{\max }$, the sail must provide a propelling thrust as long as the sailcraft moves away from the Sun and that the thrust must be set to zero when the sailcraft approaches the Sun. The opposite happens when $F_{n}=F_{n}^{\min }$ (the thrust is set to zero when the sailcraft moves away and is set on when it approaches the Sun). Note that Eq. (29) is a special case of the optimal control law for both an ideal sail model (Sauer, 1976), and a solar sail with optical force model (Dachwald et al., 2007; Mengali and Quarta, 2005b,c; Dachwald et al., 2006a).

\section{Mission applications}

Having found the steering law that either maximizes or minimizes $F_{n}$, it is now possible to apply the above results for analyzing some specific mission scenarios. 
4.1 Minimum performance to escape

As a first example, consider an escape mission from the Solar System. For a given switching strategy, that is, for a given value of $F_{n}$, the problem is to find the value of lightness number $\beta=\beta_{\text {esc }}$, that allows a sailcraft to reach a parabolic orbit within $n$ maneuvers. The condition $a_{n} \rightarrow \infty$ (parabolic orbit) corresponds to the value of $\beta$ such that the denominator of Eq. (14) vanishes, viz.

$$
\beta_{\mathrm{esc}} \triangleq \frac{1}{2 a_{0} F_{n}}
$$

Clearly, for a given $n$, the value of $\beta_{\text {esc }}$ corresponds to $F_{n}=F_{n}^{\max }$. Also note that the minimum value of $\beta$ to reach an escape condition is obtained when the last trajectory arc is a propelled arc (i.e., $n$ is odd). In fact, as previously stated, a P-C maneuver produces a decrease of the semimajor axis. Therefore, the fulfilment of the condition $a_{n} \rightarrow \infty$ with a minimum value of $\beta$ takes place necessarily when the semimajor axis increases, that is, with a C-P maneuver.

The optimal control law (29) implies that a C-P maneuver is performed at the orbit perihelion, while a P-C maneuver occurs at the orbit aphelion. Therefore, according to McInnes (2003), the optimal sequence of maneuvering points $\nu_{i}=\nu_{i}^{\star}$ is:

$$
\nu_{i}^{\star}=\left\{\begin{array}{ll}
0 & \text { if } i \text { is even } \\
\pi & \text { if } i \text { is odd }
\end{array} \quad \text { for } \quad i=0,1, \ldots,(n-1)\right.
$$

From Eq. (18), the maximum value of $F_{k}$, or $F_{k}^{\max } \triangleq F_{k}\left(\nu_{i}^{\star}\right)$, is:

$$
F_{k}^{\max }=\sum_{i=0}^{k-1} \frac{(-1)^{i}}{a_{i}\left[1-(-1)^{i} e_{i}\right]}
$$

where $a_{i}$ and $e_{i}$ are obtained from Eqs. (14) and (16) by symbolically substituting $k$ with $i$. For example, assuming $k=4$, a recursive application of Eqs. (14), (16) and (33) yields

$$
\begin{aligned}
a_{1}=\frac{p_{0}(1-\beta)}{\left(1+e_{0}\right)\left(1-e_{0}-2 \beta\right)} & , & a_{2}=\frac{p_{0}}{\left(1-e_{0}-2 \beta\right)\left(1+e_{0}+2 \beta\right)} \\
a_{3}=\frac{p_{0}(1-\beta)}{\left(1+e_{0}+2 \beta\right)\left(1-e_{0}-4 \beta\right)} & \quad & a_{4}=\frac{p_{0}}{\left(1-e_{0}-4 \beta\right)\left(1+e_{0}+4 \beta\right)}
\end{aligned}
$$


and

$$
e_{1}=\frac{\beta+e_{0}}{1-\beta} \quad, \quad e_{2}=e_{0}+2 \beta \quad, \quad e_{3}=\frac{3 \beta+e_{0}}{1-\beta} \quad, \quad e_{4}=e_{0}+4 \beta
$$

The particular structure of the above equations provides an immediate generalization to a generic value of $k \geq 1$. The result is:

$$
a_{k}= \begin{cases}\frac{p_{0}(1-\beta)}{(1-\beta)^{2}-\left(e_{0}+k \beta\right)^{2}} & \text { if } k \text { is odd } \\ \frac{p_{0}}{1-\left(e_{0}+k \beta\right)^{2}} & \text { if } k \text { is even }\end{cases}
$$

and

$$
e_{k}=\left\{\begin{array}{cl}
\frac{e_{0}+k \beta}{1-\beta} & \text { if } k \text { is odd } \\
e_{0}+k \beta & \text { if } k \text { is even }
\end{array}\right.
$$

In addition, the recursive substitution of Eqs. (36) and (37) into Eq. (33) yields

$$
F_{k}^{\max }= \begin{cases}\frac{k\left(2 e_{0}+k \beta\right)+2-\beta}{2 a_{0}\left(1-e_{0}^{2}\right)} & \text { if } k \text { is odd } \\ \frac{k\left(2 e_{0}+k \beta\right)}{2 a_{0}\left(1-e_{0}^{2}\right)} & \text { if } k \text { is even }\end{cases}
$$

The minimum lightness number $\beta_{\mathrm{esc}}^{\star} \triangleq \min \left(\beta_{\mathrm{esc}}\right)$ in a trajectory constituted by $n$ arcs, is found by substituting Eq. (38) (with $k=n, \beta=\beta_{\text {esc }}$ and $n$ odd) into Eq. (31) and solving for $\beta_{\text {esc }}$ The result is

$$
\beta_{\mathrm{esc}}^{\star}=\frac{1-e_{0}}{n+1}
$$

Equation (39) links in a simple and effective way the minimum sailcraft performance required to reach an escape condition with the eccentricity of the parking orbit $e_{0}$ and the number of trajectory arcs $n$. Note that the same result can be found also from Eq. (36) (with $k=n$ and $n$ odd), by setting the denominator to zero.

The lack of dependence on $a_{0}$ in Eq. (39) implies that the minimum performance is related to the orbit shape only. This extends the results found by Mengali and Quarta (2009a) for a spacecraft subjected to 
a constant radial thrust. Finally, note that for a circular parking orbit $\left(e_{0}=0\right)$ and a single propelled arc $(n=1)$, Eq. (39) provides $\beta_{\mathrm{esc}}^{\star}=1 / 2$, in accordance to the solution originally found by McInnes (1999).

The presence of $n$ in the denominator of Eq. (39) suggests the possibility of a reduction of $\beta_{\text {esc }}$ at will, by simply increasing the number of trajectory arcs. Although this is in principle true, there are, however, a couple of constraints that must be taken into account. On one side an increase of $n$ implies a corresponding increase in the total flight time $\Delta t_{\mathrm{esc}}$, that is, the time interval between the first and the last maneuver. In fact, $\Delta t_{\text {esc }}$ is simply equal to the sum of the half-orbital periods $\Delta t_{k}$, with $k=1,2, \ldots,(n-1)$, of the elliptic arcs that constitute the trajectory between the first and the last maneuver. Accordingly:

$$
\Delta t_{\mathrm{esc}}=\sum_{k=1}^{n-1} \Delta t_{k}=\pi \sum_{k=1}^{n-1} \sqrt{\frac{a_{k}^{3}}{\mu_{\odot}}}
$$

where $a_{k}$ is given by Eq. (36).

In addition, an increase of $n$ tends to reduce the minimum perihelion distance from the Sun. In fact, the perihelion of any arc is $r_{p_{k}}=p_{k} /\left(1+e_{k}\right)$, and from Eqs. (15) and (37) it may be verified that the perihelion distance decreases monotonically as long as $k$ is increased. Therefore, the total trajectory perihelion distance is obtained in correspondence of the last C-P maneuver (that is, when $k=n-1$ and $n$ is odd). The result is

$$
r_{p}=\frac{p_{0}}{\beta(n-1)+1+e_{0}}
$$

Note that, when $\beta=\beta_{\mathrm{esc}}^{\star}$, Eq. (41) yields

$$
r_{p}=\frac{p_{0}(n+1)}{2\left(n+e_{0}\right)}
$$

In particular, in the limit as $n \rightarrow \infty$, the perihelion radius reduces to

$$
\lim _{n \rightarrow \infty} r_{p}=\frac{p_{0}}{2} \triangleq r_{p_{\min }}
$$

which corresponds to the minimum theoretical value of perihelion distance reachable during an optimal escape trajectory. 
The practical consequence of Eq. (42) is that there exists an explicit relationship between the number of $\operatorname{arcs} n$ and the maximum temperature experienced by the sail during the mission (Rowe et al., 1978; Stimpson et al., 1978). In fact, using the model discussed in Mengali and Quarta (2009b) and Dachwald et al. (2006b), the maximum sail equilibrium temperature is given by

$$
\Theta_{\max }=\widetilde{\Theta} \sqrt{\frac{r_{\oplus}}{r_{p}}}
$$

where $r_{\oplus} \triangleq 1 \mathrm{AU}$ and $\widetilde{\Theta} \triangleq 263.56 \mathrm{~K}$ is a reference temperature depending on the optical properties of the reflecting material (McInnes, 1999). Because during the mission the sail temperature cannot exceed a given sail film maximum tolerable limit $\Theta_{\text {lim }}$ (Mengali and Quarta, 2009b), upon combining Eqs. (42) and (44) one obtains the following relationship involving an upper limit on $n$ :

$$
n \leq\lfloor f\rfloor \quad \text { with } \quad f \triangleq \frac{2 r_{\oplus} e_{0} \widetilde{\Theta}^{2}-p_{0} \Theta_{\lim }^{2}}{p_{0} \Theta_{\lim }^{2}-2 r_{\oplus} \widetilde{\Theta}^{2}}
$$

where $\lfloor\cdot\rfloor$ is the floor function.

Recall that the previous expressions for the total flight time, the minimum perihelion distance, and the maximum sail temperature are all obtained under the assumption of minimum value of lightness number necessary to reach an escape condition. Accordingly, $\Delta t_{\mathrm{esc}}, r_{p}$ and $\Theta_{\max }$ can be thought of as being the outputs of the optimization process whose performance index is $\beta$. In the following example Eqs. (40), (42), and (44) will be used to check that the simulation results meet the typical mission constraints.

Assuming a parking orbit equal to the Earth's heliocentric orbit, that is, $a_{0}=a_{\oplus} \triangleq 1 \mathrm{AU}$ and $e_{0}=e_{\oplus} \triangleq 0.01671$, with the aid of the previous relationships the results summarized in Table 1 are obtained. In this and all of the examples discussed below the total mission time is constrained to not exceed 15 years.

The information summarized in Table 1 allows one to find a compromise solution between the flight time and the minimum lightness number required to obtain an escape from the Solar System with a sailcraft deployment on a parabolic Earth-escape trajectory, that is, with zero hyperbolic excess energy with respect to the planet. Using a limit temperature $\Theta_{\lim }=513.15 \mathrm{~K}$ (Dachwald et al., 2006b), the temperature constraint does not affect the sailcraft trajectory. In fact, from Eq. (43) the minimum peri- 


\begin{tabular}{cccccc}
\hline \hline$n$ & $\beta_{\mathrm{esc}}^{\star}$ & $\begin{array}{c}a_{c} \\
{\left[\mathrm{~mm} / \mathrm{s}^{2}\right]}\end{array}$ & $\begin{array}{c}r_{p} \\
{[\mathrm{AU}]}\end{array}$ & $\begin{array}{c}\Theta_{\max } \\
{[\mathrm{K}]}\end{array}$ & $\begin{array}{c}\Delta t_{\mathrm{esc}} \\
{[\text { years }]}\end{array}$ \\
\hline 1 & 0.4916 & 2.9155 & 0.9833 & 265.7901 & 0 \\
3 & 0.2458 & 1.4577 & 0.6628 & 323.7367 & 1.8492 \\
5 & 0.1639 & 0.9718 & 0.5978 & 340.8702 & 4.0323 \\
7 & 0.1229 & 0.7289 & 0.5699 & 349.1218 & 6.6170 \\
9 & 0.0983 & 0.5831 & 0.5544 & 353.9804 & 9.5586 \\
11 & 0.0819 & 0.4859 & 0.5445 & 357.1828 & 12.8209 \\
\hline \hline
\end{tabular}

Table 1 Optimal performance for a Solar System escape starting from an Earth's parking orbit.

helion orbit is always greater than $p_{0} / 2=0.5 \mathrm{AU}$. The only active constraint is therefore the maximum time necessary to reach an escape condition. Assuming for example $\Delta t_{\text {esc }}<2$ years, from Table 1 one concludes that $n=3$ and the minimum lightness number is $\beta_{\mathrm{esc}}^{\star} \simeq 0.2458$. This result has been confirmed by numerical simulations, by integrating the equations of motion with the optimal control law (29) in the form:

$$
a_{r}= \begin{cases}\beta_{\mathrm{esc}}^{\star} \frac{\mu_{\odot}}{r^{2}} & \text { if } \dot{r} \geq 0 \\ 0 & \text { if } \dot{r}<0\end{cases}
$$

Note, in passing, that Eq. (46) coincides with the locally optimal control law, that is, the control law that maximizes the time derivative of the specific mechanical energy (Macdonald and McInnes, 2005; Macdonald et al., 2007; Mengali and Quarta, 2004, 2005a). The simulation results for $n=3$ have been summarized in Fig. 3. In the figure the specific mechanical energy $\mathcal{E}$ (which is made dimensionless with its initial value $\left.\mathcal{E}_{0} \triangleq-\mu_{\odot} /\left(2 a_{0}\right)\right)$ during the propelled arcs is calculated by taking into account the reduced gravitational parameter, that is:

$$
\mathcal{E}= \begin{cases}\frac{v^{2}}{2}-\frac{\widetilde{\mu}_{\odot}}{r} & \text { when } \dot{r} \geq 0 \\ \frac{v^{2}}{2}-\frac{\mu_{\odot}}{r} & \text { when } \dot{r}<0\end{cases}
$$

where $v$ is the sailcraft velocity magnitude. The specific mechanical energy, which has a stepwise behaviour, increases during any C-P maneuver. Note the consistency between the results of Table 1 and those 

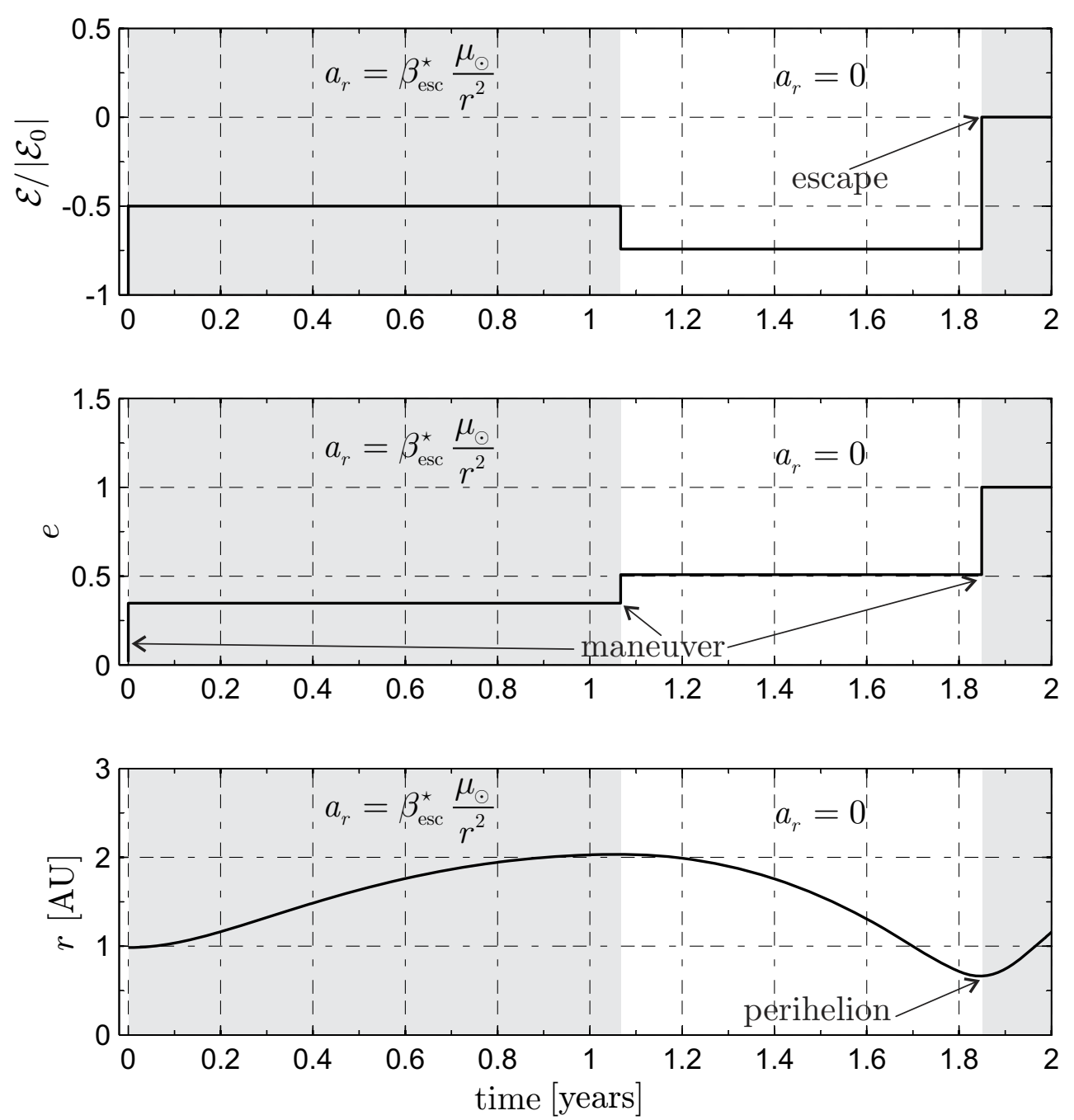

Fig. 3 Simulation results for a Solar System escape mission with $n=3, a_{0}=a_{\oplus}$ and $e_{0}=e_{\oplus}$ (grey regions correspond to propelled arcs).

shown in Fig. 3 regarding the flight time ( $\Delta t_{\mathrm{esc}} \simeq 1.849$ years $)$ and the perihelion radius $\left(r_{p} \simeq 0.66 \mathrm{AU}\right)$. The sailcraft trajectory is actually a sequence of conic arcs, as shown in Fig. 4.

As a second example, consider now the escape problem from the heliocentric Mercury's orbit $\left(a_{0}=\right.$ $a_{\Varangle} \simeq 0.387 \mathrm{AU}$ and $e_{0}=e_{\Varangle} \simeq 0.2056$ ). This example allows one to investigate the impact of the starting orbit eccentricity and the perihelion distance on the mission performance. However, we explicitly maintain that a direct comparison with the previous example (escape from an Earth's parking orbit) is not possible, as the following results do not take into account the transfer time from Earth's to Mercury's orbit.

From Table 2, the optimal escape strategy requires a single maneuver $(n=1)$, otherwise the sail film would be exposed to a temperature greater than the limit temperature of $513.15 \mathrm{~K}$. For example, assuming $n=11$, the minimum lightness number required to reach an escape condition is $\beta_{\mathrm{esc}}^{\star} \simeq 0.066$ only, see 


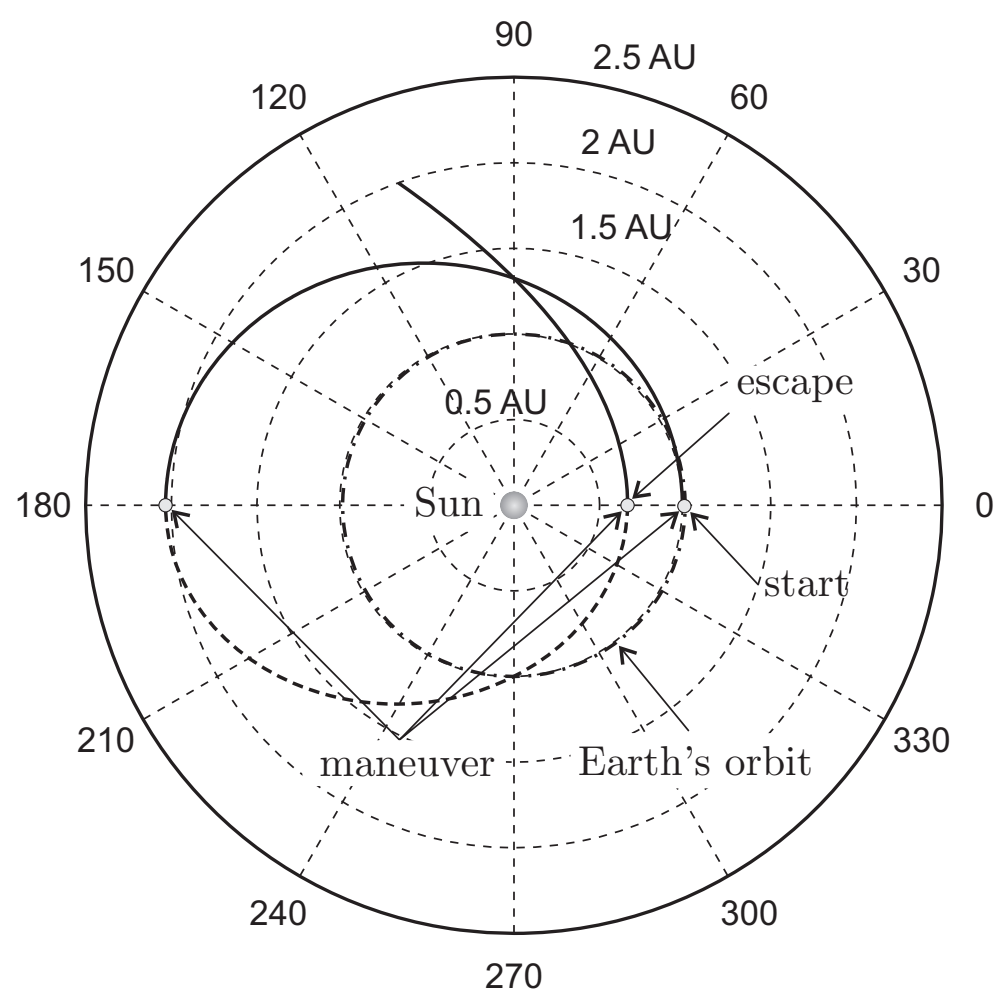

Fig. 4 Solar System escape trajectory from an Earth's orbit with $n=3$ and $\beta=\beta_{\text {esc }}^{\star}=0.2458$ (coasting arcs in dotted line, and propelled arcs in solid line).

\begin{tabular}{cccccc}
\hline \hline$n$ & $\beta_{\mathrm{esc}}^{\star}$ & $\begin{array}{c}a_{c} \\
{\left[\mathrm{~mm} / \mathrm{s}^{2}\right]}\end{array}$ & $\begin{array}{c}r_{p} \\
{[\mathrm{AU}]}\end{array}$ & $\begin{array}{c}\Theta_{\max } \\
{[\mathrm{K}]}\end{array}$ & $\begin{array}{c}\Delta t_{\mathrm{esc}} \\
{[\text { years }]}\end{array}$ \\
\hline 1 & 0.3972 & 2.3553 & 0.3075 & 475.2 & 0 \\
3 & 0.1986 & 1.1777 & 0.2313 & 548.0 & 0.4952 \\
5 & 0.1324 & 0.7851 & 0.2137 & 570.1 & 1.1216 \\
7 & 0.0993 & 0.5888 & 0.2058 & 580.9 & 1.8743 \\
9 & 0.0794 & 0.4711 & 0.2014 & 587.3 & 2.7379 \\
11 & 0.0662 & 0.3926 & 0.1985 & 591.5 & 3.7012 \\
13 & 0.0567 & 0.3365 & 0.1965 & 594.5 & 4.7553 \\
15 & 0.0496 & 0.2944 & 0.1950 & 596.7 & 5.8937 \\
17 & 0.0441 & 0.2617 & 0.1939 & 598.5 & 7.1107 \\
19 & 0.0397 & 0.2355 & 0.1930 & 599.8 & 8.4019 \\
21 & 0.0361 & 0.2141 & 0.1923 & 601.0 & 9.7633 \\
23 & 0.0331 & 0.1963 & 0.1917 & 601.9 & 11.1918 \\
25 & 0.0306 & 0.1812 & 0.1912 & 602.7 & 12.6844 \\
27 & 0.0284 & 0.1682 & 0.1908 & 603.4 & 14.2386 \\
\hline \hline
\end{tabular}

Table 2 Optimal performances for a Solar System escape starting from the Mercury's heliocentric orbit.

Eq. (39), and the total flight time is about 3.7 years. However, the corresponding maximum temperature is $\Theta_{\max } \simeq 591.5 \mathrm{~K}$ and the resulting trajectory is rather complex, as shown in Fig. 5. Note that, as a 
consequence of Eq. (15), for all of the conic arcs the semilatus rectum is constant and, accordingly, all the arcs intersect at two points.

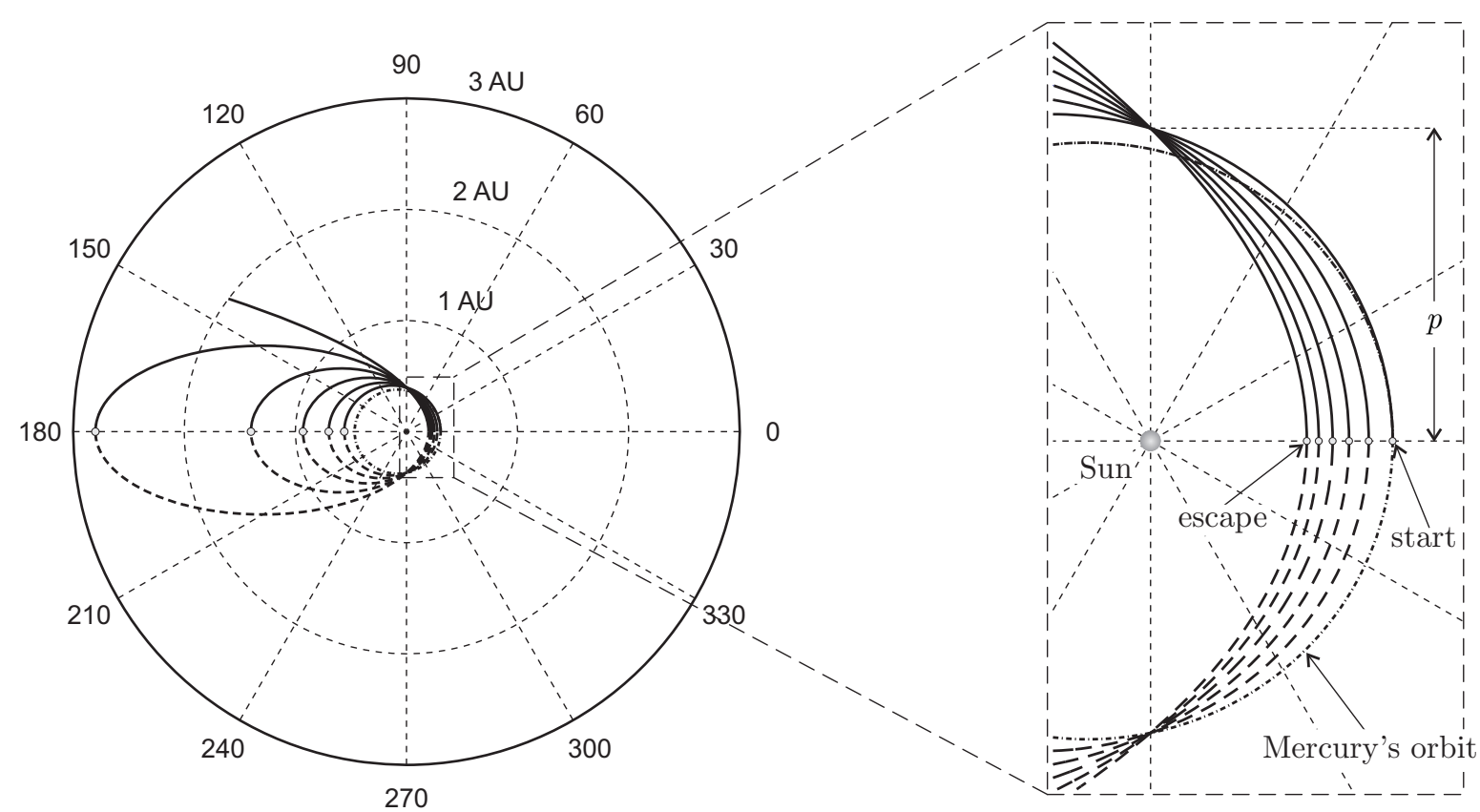

Fig. 5 Solar System escape trajectory from Mercury's orbit with $n=11$ and $\beta=\beta_{\mathrm{esc}}^{\star}=0.066$ (coasting arcs in dotted line, and propelled arcs in solid line).

As previously pointed out, an increase of the number of trajectory arcs implies a decrease of both the minimum perihelion distance and the minimum characteristic acceleration required to attain an escape condition. Such a behavior is essentially due to the substantial increase of radial acceleration that a sailcraft experiences when it approaches the Sun. For comparative purposes, it is possible to calculate the performance of a CRT spacecraft with constant thrust using the mathematical model discussed in Quarta and Mengali (2009). It can be verified that, the total flight time being the same, the characteristic acceleration required by a solar sail is much smaller than that of a CRT spacecraft. For example, assuming a flight time of 3.7012 years, a CRT spacecraft requires a characteristic acceleration $a_{c}=1.162 \mathrm{~mm} / \mathrm{s}^{2}$ to reach an escape condition, against about $0.4 \mathrm{~mm} / \mathrm{s}^{2}$ of a solar sail spacecraft, see Tab. 2 .

4.2 Minimum performance to reach a given ballistic orbit

As a second scenario, consider now a mission whose aim is to reach a final Keplerian orbit with a given (finite) value of semimajor axis $a_{f}>0$, starting from a parking orbit with semimajor axis $a_{0}$ and 
eccentricity $e_{0} \in[0,1)$. The problem here is to find the minimum value of $\beta$ required to accomplish such a mission. Recall from Eqs. (14) and (16) that the final orbit eccentricity is univocally obtained as a function of $a_{f}$. Assume that the (final) $n$-th trajectory arc starts with a P-C maneuver and that the sailcraft, once reached the final orbit, jettisons the propulsion system. This scenario corresponds to a transfer orbit constituted by an even number $n$ of elliptical arcs. Recalling from Eq. (15) that $p_{f}=p_{0}$, the final eccentricity is

$$
e_{f}=\sqrt{1-\frac{p_{0}}{a_{f}}}
$$

Therefore, the final aphelion distance $r_{a}$ is:

$$
r_{a}=a_{f}\left(1+\sqrt{1-\frac{p_{0}}{a_{f}}}\right)
$$

Note that $r_{a}$ increases monotonically with increasing $a_{f}$. As a result, the minimum lightness number necessary to obtain a given $a_{f}$ corresponds to the minimum value required to reach a given aphelion distance $r_{a}$. In particular, Eq. (49) can be used to express $a_{f}$ as a function of $r_{a}$ in a dimensionless form. In fact, solving Eq. (49) for $a_{f}$, yields:

$$
\frac{a_{f}}{p_{0}}=\frac{\left(r_{a} / p_{0}\right)^{2}}{2 r_{a} / p_{0}-1}
$$

The value of $\beta$ required to fulfil the sailcraft transfer is obtained from Eq. (14) by setting $a_{n}=a_{f}$ and choosing an even value of $n$. The result is:

$$
\beta_{a_{f}}=\frac{1-a_{0} / a_{f}}{2 a_{0} F_{n}}
$$

As in the previous escape case, the lightness number is a function of $F_{n}$ and, therefore, it depends on the choice of the $n$ control parameters $\nu_{i}$, see Eq. (18). Since the lightness number is, by definition, a positive parameter, from Eq. (51) the minimum (superscript $\star$ ) lightness number $\beta_{a_{f}}$ necessary to complete the transfer within $n$ arcs is

$$
\beta_{a_{f}}^{\star}=\left\{\begin{array}{lll}
\frac{1-a_{0} / a_{f}}{2 a_{0} F_{n}^{\max }} & \text { if } & a_{f}>a_{0} \\
\frac{1-a_{0} / a_{f}}{2 a_{0} F_{n}^{\min }} & \text { if } & a_{f}<a_{0}
\end{array}\right.
$$


where $F_{n}^{\max }$ is given by Eq. (38) with $k=n$. Also recall that $F_{n}^{\min }$ corresponds to the minimum value of $F_{n}$ with respect to the $n$ control parameters $\nu_{i}$.

The expression for $F_{n}^{\min }$ is found by selecting the perihelion distance for a P-C maneuver and, viceversa, the aphelion for a C-P maneuver, see Eq. (18). Such a control law, can be thought of equivalent to the symmetric counterpart of the previous strategy required to obtain $F_{n}^{\max }$. Using the same approach as that employed to obtain $F_{n}^{\max }$ [see Eq. (38)], the expression of $F_{n}^{\min }$ is found to be:

$$
F_{n}^{\min }= \begin{cases}\frac{n\left(n \beta-2 e_{0}\right)}{2 a_{0}\left(1-e_{0}^{2}\right)} & \text { if } n \text { is even } \\ \frac{n\left(n \beta-2 e_{0}\right)+2-\beta}{2 a_{0}\left(1-e_{0}^{2}\right)} & \text { if } n \text { is odd }\end{cases}
$$

Note that, if $a_{f}<a_{0}$, Eq. (52) implies that $F_{n}^{\min }<0$. From Eq. (53) this is possible only provided that $\beta_{a_{f}}^{\star} \in\left(0,2 e_{0} / n\right)$.

Finally, substituting (38) and (53) (with $\beta=\beta_{a_{f}}^{\star}$ ) into (52) and solving for $\beta_{a_{f}}^{\star}$, yields:

$$
\beta_{a_{f}}^{\star}=\left\{\begin{array}{lll}
\frac{\sqrt{a_{f}\left(a_{f}-p_{0}\right)}-a_{f} e_{0}}{n a_{f}} & \text { if } & a_{f}>a_{0} \\
\frac{a_{f} e_{0}-\sqrt{a_{f}\left(a_{f}-p_{0}\right)}}{n a_{f}} & \text { if } & a_{f}<a_{0}
\end{array}\right.
$$

from which one concludes that the minimum value of $a_{f}$ cannot be less than $p_{0}$. In particular, if $a_{f}=p_{0}$, Eq. (48) states that the final orbit is circular with a radius equal to $p_{0}$.

If, instead, $a_{f}>a_{0}$, an increase of $n$ implies a corresponding decrease of $\beta_{a_{f}}^{\star}$, in accordance to Eq. (54). Similarly to the previously discussed case of mission escape, an increase of $n$ also increases the total mission time and decreases the perihelion distance $r_{p}$. The latter can be obtained from the following expression:

$$
r_{p}=\frac{n p_{0} a_{f}}{n a_{f}\left(1+e_{0}\right)-(n-2) a_{f} e_{0}+(n-2) \sqrt{a_{f}\left(a_{f}-p_{0}\right)}}
$$

The above results can be used to investigate, for example, the minimum performance necessary to reach a heliocentric distance equal to the semimajor axis of Mars' orbit $\left(r_{a}=a_{\mathrm{O}^{7}} \simeq 1.523 \mathrm{AU}\right.$ ) or to the semimajor axis of Jupiter's orbit $\left(r_{a}=a_{4} \simeq 5.203 \mathrm{AU}\right)$, starting from an Earth's heliocentric orbit 
$\left(a_{0}=a_{\oplus}\right.$ and $\left.e_{0}=e_{\oplus}\right)$. These examples are representative of flyby missions towards an outer planet under the assumption that both the inclination and the eccentricity of the target planet orbit are neglected. The mission performance for these two cases are summarized in Tables 3 and 4 as a function of $n$.

\begin{tabular}{cccccc}
\hline \hline$n$ & $\beta_{a_{f}}^{\star}$ & $\begin{array}{c}a_{c} \\
{\left[\mathrm{~mm} / \mathrm{s}^{2}\right]}\end{array}$ & $\begin{array}{c}r_{p} \\
{[\mathrm{AU}]}\end{array}$ & $\begin{array}{c}\Theta_{\max } \\
{[\mathrm{K}]}\end{array}$ & $\begin{array}{c}\Delta t_{a_{f}} \\
{[\text { years] }}\end{array}$ \\
\hline 2 & 0.1634 & 0.9692 & 0.9833 & 265.8 & 0.7669 \\
4 & 0.0817 & 0.4846 & 0.8471 & 286.3 & 1.8013 \\
6 & 0.0545 & 0.3231 & 0.8097 & 292.8 & 2.8584 \\
8 & 0.0409 & 0.2423 & 0.7923 & 296.1 & 3.9209 \\
10 & 0.0327 & 0.1938 & 0.7821 & 298.0 & 4.9855 \\
12 & 0.0272 & 0.1615 & 0.7755 & 299.2 & 6.0512 \\
14 & 0.0233 & 0.1385 & 0.7709 & 300.1 & 7.1174 \\
16 & 0.0204 & 0.1211 & 0.7674 & 300.8 & 8.1840 \\
18 & 0.0182 & 0.1077 & 0.7647 & 301.3 & 9.2508 \\
20 & 0.0163 & 0.0969 & 0.7626 & 301.8 & 10.3179 \\
22 & 0.0149 & 0.0881 & 0.7609 & 302.1 & 11.3850 \\
24 & 0.0136 & 0.0808 & 0.7595 & 302.4 & 12.4522 \\
26 & 0.0126 & 0.0746 & 0.7583 & 302.6 & 13.5195 \\
28 & 0.0117 & 0.0692 & 0.7572 & 302.8 & 14.5869 \\
\hline \hline
\end{tabular}

Table 3 Optimal performances for a Mars flyby starting from an Earth's heliocentric orbit $\left(r_{a}=1.523 \mathrm{AU}\right.$ and $a_{f}=$ $1.133 \mathrm{AU})$.

\begin{tabular}{cccccc}
\hline \hline$n$ & $\beta_{a_{f}}^{\star}$ & $\begin{array}{c}a_{c} \\
{\left[\mathrm{~mm} / \mathrm{s}^{2}\right]}\end{array}$ & $\begin{array}{c}r_{p} \\
{[\mathrm{AU}]}\end{array}$ & $\begin{array}{c}\Theta_{\max } \\
{[\mathrm{K}]}\end{array}$ & $\begin{array}{c}\Delta t_{a_{f}} \\
{[\text { years }]}\end{array}$ \\
\hline 2 & 0.3956 & 2.3457 & 0.9833 & 265.8 & 3.4986 \\
4 & 0.1978 & 1.1729 & 0.7079 & 313.2 & 4.3653 \\
6 & 0.1319 & 0.7819 & 0.6474 & 327.5 & 5.7625 \\
8 & 0.0989 & 0.5864 & 0.6209 & 334.4 & 7.2993 \\
10 & 0.0791 & 0.4691 & 0.6060 & 338.5 & 8.8985 \\
12 & 0.0659 & 0.3910 & 0.5965 & 341.2 & 10.5317 \\
14 & 0.0565 & 0.3351 & 0.5899 & 343.1 & 12.1856 \\
16 & 0.0494 & 0.2932 & 0.5850 & 344.5 & 13.8528 \\
\hline \hline
\end{tabular}

Table 4 Optimal performances for a Jupiter flyby starting from an Earth's heliocentric orbit $\left(r_{a}=5.203 \mathrm{AU}\right.$ and $a_{f}=$ $2.878 \mathrm{AU})$.

The tables show that although in both cases the minimum perihelion distance is tolerable, unfortunately the flight times for a mission to Jupiter are rather long. In fact the optimal trajectory (that is, with 
a minimum value of $\beta$ ) when $n=2$ needs a flight time of about $\Delta t_{a_{f}}=3.5$ years with a lightness number of $\beta_{a_{f}}^{\star}=0.395\left(a_{c} \simeq 2.34 \mathrm{~mm} / \mathrm{s}^{2}\right)$. The Mars case is much more favorable, as the flight time with $n=2$ is 0.77 years only, and a moderate lightness number equal to $\beta_{a_{f}}^{\star}=0.1634\left(a_{c} \simeq 0.97 \mathrm{~mm} / \mathrm{s}^{2}\right)$ is necessary. The optimal trajectory for a Mars flyby, with $n=2$ and $n=8$ is shown in Fig. 6 . Note that if $n=2$, see Fig. 6(a), the sailcraft transfer is close to a more familiar circle-to-circle Hohmann transfer (recall that $\left.e_{\oplus} \ll 1\right)$. According to McInnes (2003), when $n=2$ the sailcraft trajectory shows one propelled arc only, which connects the initial and final circular orbits in a quasi-Hohmann fashion. In fact, from Eqs. (50) and (54), the corresponding lightness number $\beta_{a_{f}}^{\star} \simeq\left(1-a_{0} / a_{f}\right) / 2$ is the minimum value required to put the aphelion of the transfer trajectory on the target circular orbit. This corresponds to obtaining, at the end of the last propelled arc, a flyby with hyperbolic excess speed $v_{\infty}$ (with respect to the target planet) given by:

$$
v_{\infty}=\sqrt{\frac{\mu_{\odot}}{r_{a}}}-\frac{\sqrt{\mu_{\odot} p_{0}}}{r_{a}}
$$

When the number of arcs increases, see Fig. 6(b) where $n=8$, the transfer trajectory becomes more involved but the aphelion still lies on the final target orbit. Note that the change from $n=2$ to $n=8$ in an Earth-Mars flyby mission, entails a saving of about $75 \%$ in the lightness number and an increase of $410 \%$ in the flight time, see Table 3 . The same reduction in $\beta_{a_{f}}^{\star}(75 \%)$ occurs in an Earth-Jupiter flyby mission, see Figure 7, when the strategy switches from $n=2$ to $n=8$, whereas the flight time doubles.

A further interesting employment of the above theory is given by the creation of an orbital resonance with the departure orbit. Resonant orbits can be used for scientific purposes or to generate multiple flyby maneuvers with Earth. An example is shown in Table 5, which summarizes the optimal performance required to reach a 1:2 resonant orbit, having a semimajor axis equal to $a_{f}=\sqrt[3]{4} a_{\oplus}=1.5874 \mathrm{AU}$. The resulting resonant orbit and the transfer trajectory towards that orbit are illustrated in Fig. 8.

\section{Conclusions}

The dynamics of a sailcraft subjected to a modulated radial thrust, whose magnitude varies as the inverse square distance from the primary body, have been thoroughly studied. When the thrust is on, the sailcraft experiences a gravitational force whose magnitude is reduced with respect to the local solar 


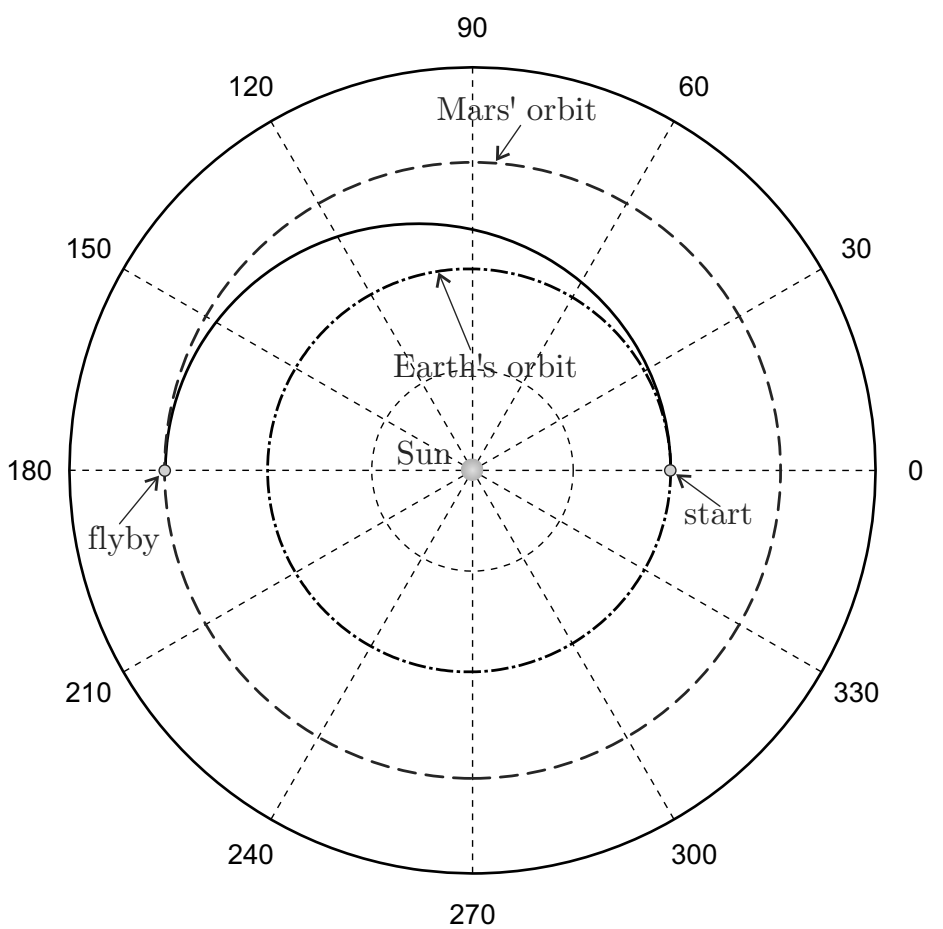

(a) $n=2, \beta=\beta_{a_{f}}^{\star}=0.1634$.

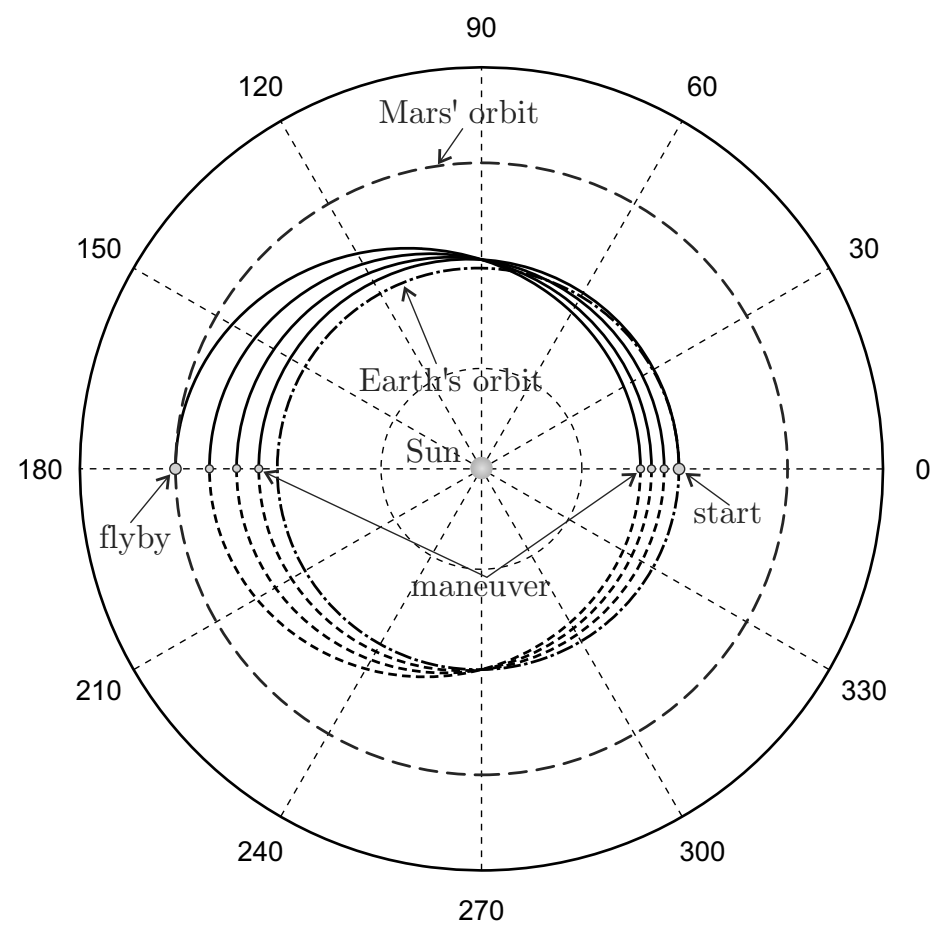

(b) $n=8, \beta=\beta_{a_{f}}^{\star}=0.0409$

Fig. 6 Optimal trajectory for a Mars flyby (coasting arcs in dotted line, and propelled arcs in solid line). 


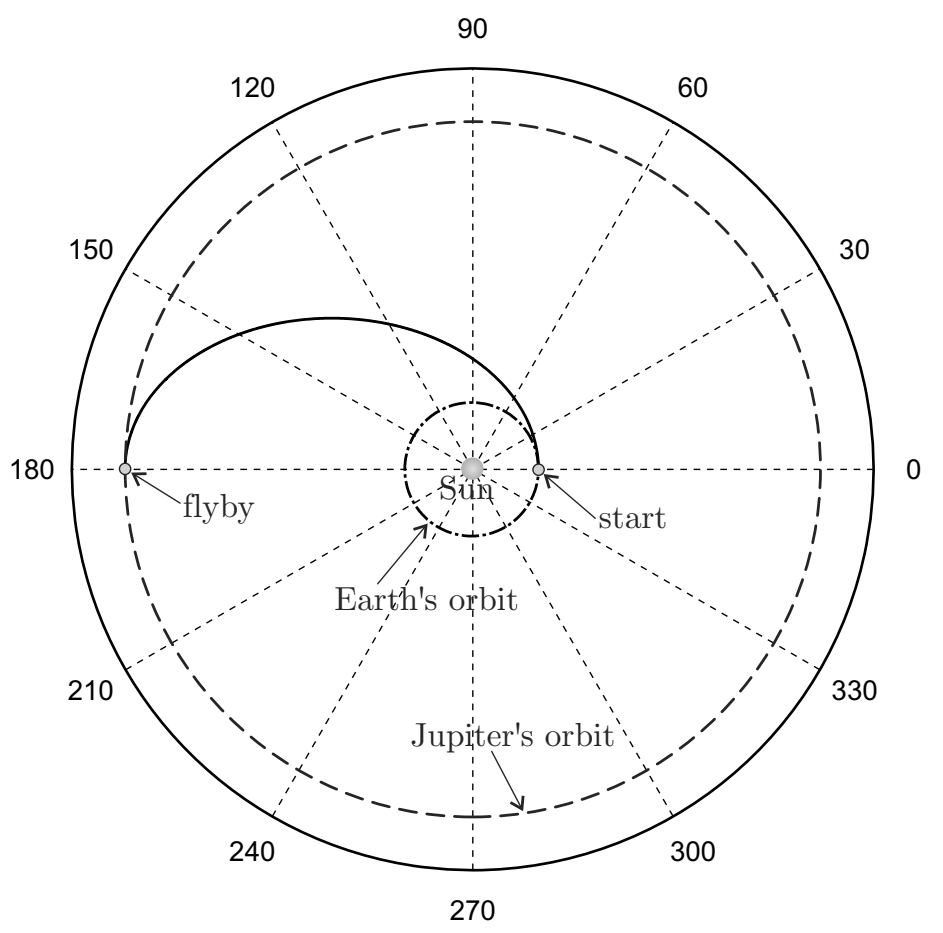

(a) $n=2, \beta=\beta_{a_{f}}^{\star}=0.3956$.

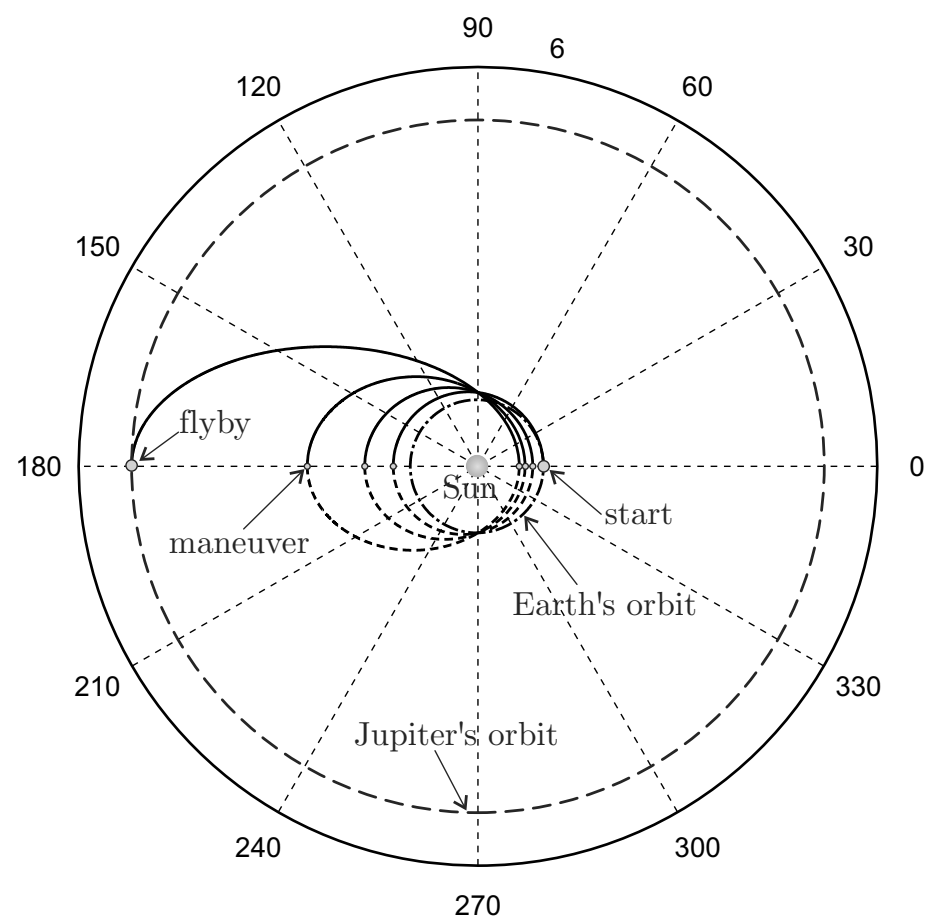

(b) $n=8, \beta=\beta_{a_{f}}^{\star}=0.0989$

Fig. 7 Optimal trajectory for a Jupiter flyby (coasting arcs in dotted line, and propelled arcs in solid line). 


\begin{tabular}{cccccc}
\hline \hline$n$ & $\beta_{a_{f}}^{\star}$ & $a_{c}$ & $r_{p}$ & $\begin{array}{c}\Theta_{\max } \\
{\left[\mathrm{mm} / \mathrm{s}^{2}\right]}\end{array}$ & $\begin{array}{c}\Delta t_{a_{f}} \\
{[\mathrm{AU}]}\end{array}$ \\
{$[\mathrm{K}]$} & {$[$ years] } \\
\hline 2 & 0.2959 & 1.7545 & 0.9833 & 265.7901 & 1.4011 \\
4 & 0.1479 & 0.8773 & 0.7616 & 301.9975 & 2.4678 \\
6 & 0.0986 & 0.5848 & 0.7084 & 313.1376 & 3.6689 \\
8 & 0.0740 & 0.4386 & 0.6845 & 318.5617 & 4.9029 \\
10 & 0.0592 & 0.3509 & 0.6709 & 321.7722 & 6.1501 \\
12 & 0.0493 & 0.2924 & 0.6621 & 323.8949 & 7.4038 \\
14 & 0.0423 & 0.2506 & 0.6560 & 325.4026 & 8.6614 \\
16 & 0.0370 & 0.2193 & 0.6515 & 326.5288 & 9.9213 \\
18 & 0.0329 & 0.1949 & 0.6480 & 327.4021 & 11.1827 \\
20 & 0.0296 & 0.1755 & 0.6453 & 328.0990 & 12.4453 \\
22 & 0.0269 & 0.1595 & 0.6430 & 328.6682 & 13.7086 \\
24 & 0.0247 & 0.1462 & 0.6412 & 329.1417 & 14.9726 \\
\hline \hline
\end{tabular}

Table 5 Optimal performance to reach a 1:2 Earth resonant orbit $\left(a_{f}=1.5874 \mathrm{AU}\right)$.

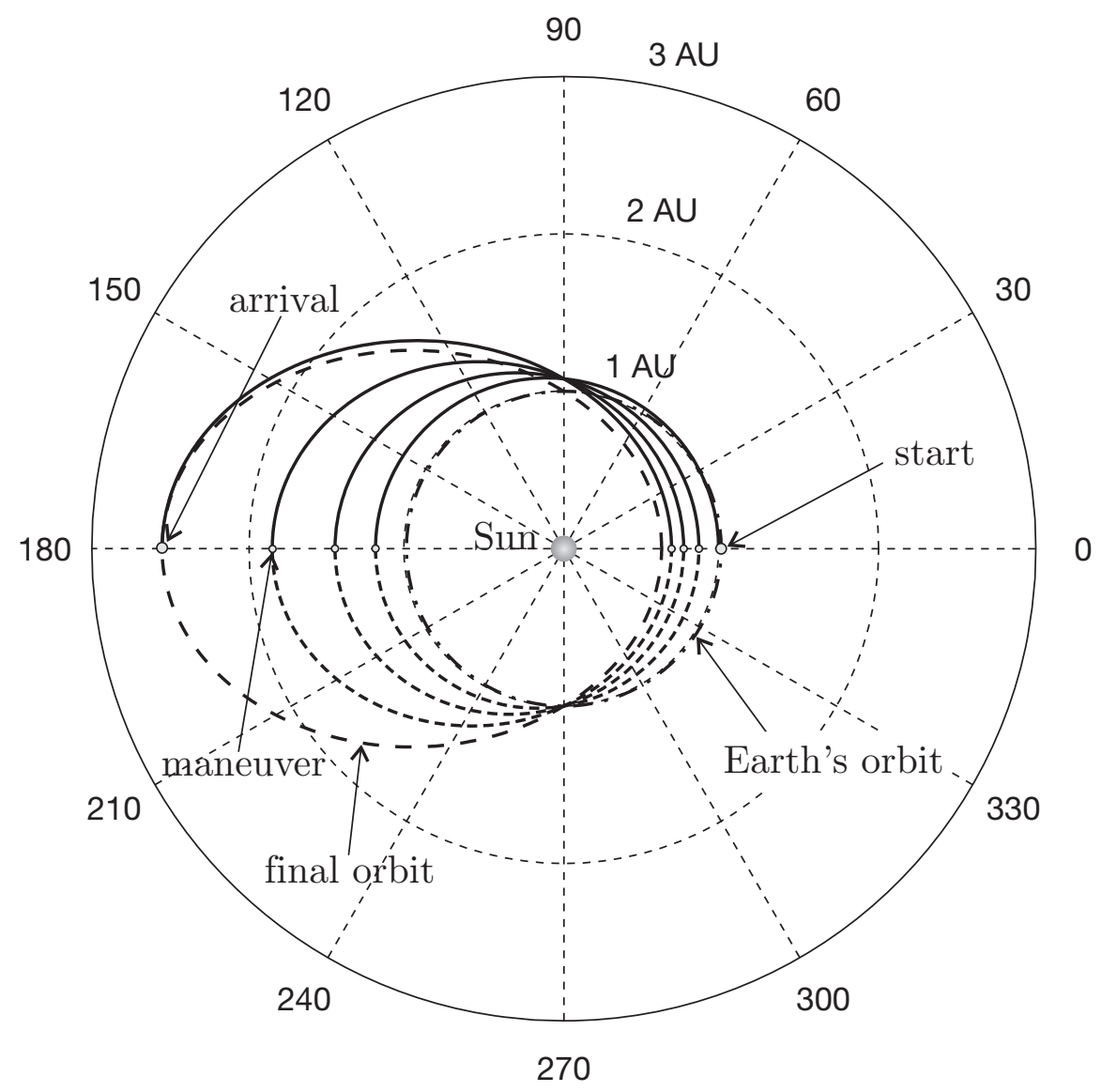

Fig. 8 Transfer trajectory to reach a 1:2 Earth resonant orbit (coasting arcs in dotted line, and propelled arcs in solid line).

gravity. As a result, the sailcraft trajectory is a sequence of either propelled or ballistic conic arcs. The trajectory analysis has been performed in an optimal framework by maximizing or minimizing a suitable 
scalar performance index. The mathematical structure of the problem guarantees the achievement of some simple analytical relationships that can be used effectively to find compromise solutions between the required maximum thrust level to accomplish a given mission and the total flight time. In particular, the above mathematical model has been applied to a solar sail spacecraft and different mission scenarios have been investigated. For the escape trajectory problem, some analytical relationships are found that link the number of trajectory arcs with the minimum required lightness number, the flight time, and the perihelion distance. In addition, the minimum performance necessary to reach a ballistic arc with a given value of semimajor axis has been discussed. The resulting steering law has been applied to study flyby missions to outer planets and to generate Earth resonant Keplerian orbits. The obtained results confirm that a modulated radial thrust is superior with respect to a continuous radial thrust strategy, and the performance improvements can be calculated analytically.

\section{Acknowledgments}

This research was financed in part by a "Paolo Manes" grant. Alessandro A. Quarta gratefully acknowledges the support of the Manes family.

\section{References}

Betts, J. T., March-April 1998. Survey of numerical methods for trajectory optimization. Journal of Guidance, Control, and Dynamics 21 (2), 193-207, doi: 10.2514/2.4231.

Boltz, F. W., May-June 1991. Orbital motion under continuous radial thrust. Journal of Guidance, Control, and Dynamics 14 (3), 667-670, doi: 10.2514/3.20690.

Bryson, A. E., Ho, Y. C., 1975. Applied Optimal Control. Hemisphere Publishing Corporation, New York, NY, Ch. 2, pp. 71-89.

Dachwald, B., Macdonald, M., McInnes, C. R., Mengali, G., Quarta, A. A., July-August 2007. Impact of optical degradation on solar sail mission performance. Journal of Spacecraft and Rockets 44 (4), 740-749, doi: $10.2514 / 1.21432$. 
Dachwald, B., Mengali, G., Quarta, A. A., Macdonald, M., September-October 2006a. Parametric model and optimal control of solar sails with optical degradation. Journal of Guidance, Control, and Dynamics 29 (5), 1170-1178, doi: 10.2514/1.20313.

Dachwald, B., Ohndorf, A., Wie, B., August 21-24 2006b. Solar sail trajectory optimization for the solar polar imager (spi) mission. In: AIAA/AAS Astrodynamics Specialist Conference and Exhibit. AIAA Paper 2006-6177, Keystone, Colorado.

Dellnitz, M., Ober-Blöbaum, S., Post, M., Schütze, O., Thiere, B., November 2009. A multi-objective approach to the design of low thrust space trajectories using optimal control. Celestial Mechanics and Dynamical Astronomy 105 (1-3), 33-59, doi: 10.1007/s10569-009-9229-y.

Kim, M., Hall, C. D., August 2005. Symmetries in the optimal control of solar sail spacecraft. Celestial Mechanics and Dynamical Astronomy 92 (4), 273-293, doi: 10.1007/s10569-004-2530-x.

Kirpichnikov, S. N., Kirpichnikova, E. S., Polyakhova, E. N., Shmyrov, A. S., September 1995. Planar heliocentric roto-translatory motion of a spacecraft with a solar sail of complex shape. Celestial Mechanics and Dynamical Astronomy 63 (3-4), 255-269, doi: 10.1007/BF00692290.

Koblik, V., Polyakhova, E., Sokolov, L., 2003. Controlled solar sail transfers into near-sun regions combined with planetary gravity-assist flybys. Celestial Mechanics and Dynamical Astronomy 86 (1), 59-80, doi: 10.1023/A:1023626917595.

Lawden, D. F., 1963. Optimal Trajectories for Space Navigation. Butterworths, London, pp. 54-68.

Macdonald, M., McInnes, C. R., September-October 2005. Analytical control laws for planet-centered solar sailing. Journal of Guidance, Control, and Dynamics 28 (5), 1038-1048, doi: 10.2514/1.11400.

Macdonald, M., McInnes, C. R., Dachwald, B., January-February 2007. Heliocentric solar sail orbit transfers with locally optimal control laws. Journal of Spacecraft and Rockets 44 (1), 273-276, doi: $10.2514 / 1.17297$.

McInnes, C. R., 1999. Solar Sailing: Technology, Dynamics and Mission Applications. Springer-Praxis Series in Space Science and Technology. Springer-Verlag, Berlin, pp. 38-40, 121-129.

McInnes, C. R., September-October 2003. Orbits in a generalized two-body problem. Journal of Guidance, Control, and Dynamics 26 (5), 743-749, doi: 10.2514/2.5129. 
McInnes, C. R., Brown, J. C., September 1990. The dynamics of solar sails with a non-point source of radiation pressure. Celestial Mechanics and Dynamical Astronomy 49 (3), 249-264, doi: 10.1007/BF00049416.

Mengali, G., Quarta, A. A., November-December 2004. Earth escape by ideal sail and solar-photon thrustor spacecraft. Journal of Guidance, Control, and Dynamics 27 (6), 1105-1108, doi: 10.2514/1.10637.

Mengali, G., Quarta, A. A., September-October 2005a. Near-optimal solar-sail orbit-raising from low earth orbit. Journal of Spacecraft and Rockets 42 (5), 954-958, doi: 10.2514/1.14184.

Mengali, G., Quarta, A. A., November-December 2005b. Optimal control laws for axially symmetric solar sails. Journal of Spacecraft and Rockets 42 (6), 1130-1133, doi: 10.2514/1.17102.

Mengali, G., Quarta, A. A., January-February 2005c. Optimal three-dimensional interplanetary rendezvous using nonideal solar sail. Journal of Guidance, Control, and Dynamics 28 (1), 173-177, doi: $10.2514 / 1.8325$.

Mengali, G., Quarta, A. A., March-April 2007. Trajectory design with hybrid low-thrust propulsion system. Journal of Guidance, Control, and Dynamics 30 (2), 419-426, doi: 10.2514/1.22433.

Mengali, G., Quarta, A. A., May-June 2009a. Escape from elliptic orbit using constant radial thrust. Journal of Guidance, Control, and Dynamics 32 (3), 1018-1022, doi: 10.2514/1.43382.

Mengali, G., Quarta, A. A., March-April 2009b. Solar sail near-optimal circular transfers with plane change. Journal of Guidance, Control, and Dynamics 32 (2), 456-463, doi: 10.2514/1.38079.

Mengali, G., Quarta, A. A., December 2009c. Solar sail trajectories with piecewise-constant steering laws. Aerospace Science and Technology 13 (8), 431-441, doi: 10.1016/j.ast.2009.06.007.

Mengali, G., Quarta, A. A., Circi, C., Dachwald, B., March-April 2007. Refined solar sail force model with mission application. Journal of Guidance, Control, and Dynamics 30 (2), 512-520, doi: 10.2514/1.24779.

Otten, M., McInnes, C. R., May-June 2001. Near minimum-time trajectories for solar sails. Journal of Guidance, Control, and Dynamics 24 (3), 632-634, doi: 10.2514/2.4758.

Quarta, A. A., Mengali, G., December 2009. Optimal switching strategy for radially accelerated trajectories. Celestial Mechanics and Dynamical Astronomy 105 (4), 361-377, doi: 10.1007/s10569-009-9233-2.

Racca, G. D., January 2003. New challenges to trajectory design by the use of electric propulsion and other new means of wandering in the solar system. Celestial Mechanics and Dynamical Astronomy 
85 (1), 1-24, doi: 10.1023/A:1021787311087.

Rowe, W. M., Luedke, E. E., Edwards, D. K., 24-26 May 1978. Thermal radiative properties of solar sail film materials. In: American Institute of Aeronautics and Astronautics and American Society of Mechanical Engineers, Thermophysics and Heat Transfer Conference, 2nd. AIAA Paper 78-852, Palo Alto, CA.

Sauer, Jr, C. G., August 18-20 1976. Optimum solar-sail interplanetary trajectories. In: AIAA/AAS Astrodynamics Conference. AIAA Paper 76-792, San Diego, CA.

Stengel, R. F., 1994. Optimal Control and Estimation. Dover Publications, Mineola, NY, pp. 222-254.

Stimpson, L. D., Greenfield, M. L., Jaworski, W., Wolf, F., May 24-26 1978. Thermal control of a solar sail. In: 2nd AIAA/ASME Thermophysics and Heat Transfer Conference. AIAA Paper 78-885, Palo Alto, CA.

Wright, J. L., 1992. Space Sailing. Gordon and Breach Science Publisher, Berlin, pp. 223-226.

Yamakawa, H., January-February 2006. Optimal radially accelerated interplanetary trajectories. Journal of Spacecraft and Rockets 43 (1), 116-120, doi: 10.2514/1.13317. 